\title{
ABSOLUTE CONTINUTTY OF EIGENVECTORS OF TIME-VARYING OPERATORS
}

\author{
ANDREW F. ACKER ${ }^{1}$
}

\begin{abstract}
If $\boldsymbol{K}(t)$ is a compact, selfadjoint operator function of a real variable $t$ with distinct eigenvalues at each $t$, we show that the eigenvalues and eigenvectors are absolutely continuous and that $\{\boldsymbol{K}(t)\}$ is a commuting set provided that $\boldsymbol{K}(t)$ commutes with its time derivative $K^{\prime}(t)$ at each $t$. The distinct eigenvalue condition is shown to be necessary.
\end{abstract}

1. Introduction. Let $\boldsymbol{H}$ be a real Hilbert space, i.e. the inner product on $\boldsymbol{H}$ is real valued. Let $\boldsymbol{K}(t): \boldsymbol{H} \rightarrow \boldsymbol{H}$ be a compact linear operator for each $t$ in a closed real interval $J$, and assume $K(t)$ is absolutely continuous in the operator norm on $J$. We will prove the following:

TheOREM 1. Assume $\lambda(t)$ is a distinct (at each $t \in J$ ), absolutely continuous eigenvalue function of $K(t)$ on $J$, and let $\varphi(t)$ be a corresponding normalized eigenvector function. Then $\varphi(t)$ can be chosen absolutely continuous on $\mathbf{J}$.

This result can be used inductively in connection with the usual spectral theory for compact, selfadjoint operators to prove the following:

THEOREM 2. Assume $\boldsymbol{K}(\boldsymbol{t})$ is selfadjoint and has only distinct, nonzero eigenvalues at each $t \in J$. Then there is a sequence of absolutely continuous (on $J$ ) eigenvalue and (corresponding, normalized) eigenvector functions: $\left\{\lambda_{n}(t), \varphi_{n}(t)\right\}_{n}$ which include the spectrum of $K(t)$ at each $t$, and

$$
K(t) x=\sum \lambda_{n}(t) \varphi_{n}(t)\left\langle\varphi_{n}(t), x\right\rangle \quad(x \in H \text { and } t \in J) .
$$

Received by the editors December 18, 1972 and, in revised form, February 16, 1973. AMS (MOS) subject classifications (1970). Primary 47-00, 47B05, 47B10, 47A55, 15-00, 15A18, $15 \mathrm{~A} 27$.

Key words and phrases. Compact operator function, eigenvalue and eigenvector functions, "real" Hilbert space, selfadjoint, distinct eigenvalues, absolute continuity, commuting set of operators.

1 This problem occurred in connection with the problem of factoring unbounded operator functions during my doctoral research at Boston University, 1971. I wish to thank Professor Marvin I. Freedman for helpful suggestions.

(c) American Mathematical Society 1974 
The results concerning continuity of eigenvalues and eigenvectors have a natural application to perturbation methods. Also Theorem 2 may be used to prove:

THEOREM 3. Let $K(t)$ be selfadjoint and have only distinct, nonzero eigenvalues in $J$. Assume $K(t)$ commutes with its derivative $K^{\prime}(t)$ almost everywhere in $J$. Then $\{\boldsymbol{K}(t) \mid t \in J\}$ is a commuting set.

All the theorems can be extended to corresponding theorems for unbounded operator functions which have a compact inverse for each $t \in J$. Theorems similar to 2 and 3 are proven for a square matrix function $K(t)$ which is not selfadjoint (and can have the eigenvalue 0) in A. Acker [1]. An example in $\$ 4$ shows the distinct eigenvalue condition is necessary in each above theorem.

2. The proof of Theorem 1. The proof is simplified by agreeing to call an operator $A$ " $\delta$-positive" (for a specified $\delta>0$ ) if $|A x| \geqq \delta|x|$ for all vectors $x$ orthogonal to the nullspace of $A$. One can check that any operator of the form: $\boldsymbol{K}-\lambda \boldsymbol{I}$, where $\boldsymbol{K}$ is compact, $\boldsymbol{I}$ is the identity, and $\lambda \neq 0$, is $\delta$-positive for some, possibly very small, positive $\delta$. If we set $A(t)=K(t)-\lambda(t) I$, then Theorem 1 can be restated as follows:

LEMMA. For each $t \in J$, let the operator $A(t): H \rightarrow H$ be $\delta(t)$-positive (where $\delta(t)>0$ ) and have 0 as a distinct eigenvalue. Assume the operator function $A(t)$ is absolutely continuous on $J$ in the operator norm. Then the corresponding (to 0$)$ normalized eigenvector function $\varphi(t)$ can be chosen absolutely continuous on $\mathrm{J}$.

Proof. At each. $t, \varphi(t)$ is unique only to a factor of \pm 1 . (Complex multiples are not in $\boldsymbol{H}$.) For a specified choice of $\varphi(t)$ and $t_{0}, t \in J$, let $p\left(t_{0}, t\right)=\left\langle\varphi\left(t_{0}\right), \varphi(t)\right\rangle \varphi\left(t_{6}\right)$ and $p_{\perp}\left(t_{0}, t\right)=\varphi(t)-p\left(t_{0}, t\right)$ so that $p_{\perp}\left(t_{0}, t\right)$ is orthogonal to $\varphi\left(t_{0}\right)$ and $\left|p\left(t_{0}, t\right)\right|^{2}+\left|p_{\perp}\left(t_{0}, t\right)\right|^{2}=1$. One can check that

$$
A\left(t_{0}\right) p_{\perp}\left(t_{0}, t\right)=\left[A\left(t_{0}\right)-A(t)\right] \varphi(t) .
$$

Since $A\left(t_{0}\right)$ is $\delta\left(t_{0}\right)$-positive and $|\varphi(t)|=1$ for all $t$, equation 1 implies (where $\|\cdot\|$ is the operator norm) that

$$
\delta\left(t_{0}\right)\left|p_{\perp}\left(t_{0}, t\right)\right| \leqq\left\|A(t)-A\left(t_{0}\right)\right\| \quad \text { for all } t .
$$

We conclude that $\left|p_{\perp}\left(t_{0}, t\right)\right| \rightarrow 0,\left|p\left(t_{0}, t\right)\right| \rightarrow 1$, and $\left|\left\langle\varphi\left(t_{0}\right), \varphi(t)\right\rangle\right| \rightarrow 1$ as $t \rightarrow t_{0}$. For any $t_{0} \in J$, there is a relatively open interval $I\left(t_{0}\right)$ containing $t_{0}$ on which $\left\langle\varphi\left(t_{0}\right), \varphi(t)\right\rangle \neq 0$. Redefine $\varphi(t)$ at each $t \in I\left(t_{0}\right)$ by multiplication by \pm 1 so that $\left\langle\varphi\left(t_{0}\right), \varphi(t)\right\rangle$ is positive. Then $\left\langle\varphi\left(t_{0}\right), \varphi(t)\right\rangle \rightarrow 1$ and $\varphi(t) \rightarrow \varphi\left(t_{0}\right)$ as $t \rightarrow t_{0}$. Let $I^{*}\left(t_{0}\right)$ be a relatively open subinterval of $I\left(t_{0}\right)$ in which $\left\langle\varphi\left(t_{0}\right), \varphi(t)\right\rangle>\frac{1}{2}$ and $\left|\varphi\left(t_{0}\right)-\varphi(t)\right|<\frac{1}{2}$. It is easily seen that 
the product $\left\langle\varphi\left(t_{1}\right), \varphi\left(t_{2}\right)\right\rangle$ is positive whenever $t_{1}$ and $t_{2}$ are in $I^{*}\left(t_{0}\right)$, and therefore the preceding argument shows that the redefined function $\varphi(t)$ is continuous throughout $I^{*}\left(t_{0}\right)$.

It is intuitive that if $\boldsymbol{A}(t)$ and $\varphi(t)$ (which spans the nullspace) are continuous, then $\boldsymbol{A}(t)$ is uniformly $\delta$-positive on a sufficiently small interval $I^{* *}\left(t_{0}\right)$ about $t_{0}$. This is proven starting with the equation:

$$
A(t) \psi=\left[A(t)-A\left(t_{0}\right)\right] \psi+A\left(t_{0}\right)\left[\psi-\left\langle\psi, \varphi\left(t_{0}\right)-\varphi(t)\right\rangle \psi^{\prime}\left(t_{0}\right)\right]
$$

which holds whenever $\psi$ is orthogonal to $\varphi(t)$.

One can show that $\left|\varphi\left(t_{1}\right)-\varphi\left(t_{2}\right)\right|<\sqrt{ } 2\left|p_{1}\left(t_{1}, t_{2}\right)\right|$ for $t_{1} ; t_{2} \in I^{*}\left(t_{0}\right)$. Use this in connection with equation 2 and the uniform $\delta$-positivity of $A(t)$ to obtain (for a fixed positive $\delta$ )

$$
\delta\left|\varphi\left(t_{1}\right)-\varphi\left(t_{2}\right)\right|<\sqrt{ } 2\left\|A\left(t_{1}\right)-A\left(t_{2}\right)\right\| \quad \text { (whenever } t_{1}, t_{2} \in I^{* *}\left(t_{0}\right) \text { ). }
$$

This immediately shows $\varphi(t)$ is absolutely continuous on $I^{* *}\left(t_{0}\right)$. The result is easily extended to the (compact) interval $J$.

3. The proof of Theorem 3. It will be shown that if $K(t)$ commutes with $K^{\prime}(t)$ (written: $K(t) \sim K^{\prime}(t)$ ) a.e. in $J$, then the absolutely continuous eigenvector functions $\varphi_{n}(t)$ of $\boldsymbol{K}(t)$ are all constant. Assume $\varphi(t)$ is the eigenvector function corresponding to the eigenvalue function $\lambda(t)$ and let $A(t)=K(t)-\lambda(t) I$. Then $A^{\prime}(t)$ exists a.e. and $A(t) \sim A^{\prime}(t)$ a.e. in $J$. Since 0 is a distinct eigenvalue of $A(t)$, we conclude that $\varphi(t)$ is also an eigenvector of $A^{\prime}(t)$, i.e. $A^{\prime}(t) \varphi(t)=\alpha(t) \varphi(t)$ for a real function $\alpha(t)$. From $A(t) \varphi(t)=0$ we obtain $A^{\prime}(t) \varphi(t)+A(t) \varphi^{\prime}(t)=0$. Therefore $A(t) \varphi^{\prime}(t)+$ $\alpha(t) \varphi(t)=0$ a.e. The vector product of this equation with $\varphi(t)$ shows that $\alpha(t)=0$ a.e. in $J$. Therefore $\varphi(t)$ and $\varphi^{\prime}(t)$ are both eigenvectors of $A(t)$ at the distinct eigenvalue 0 , and it follows that: $\varphi^{\prime}(t)=c(t) \varphi(t)$ for a real function $c(t)$. We find that $c(t)=\left\langle\varphi(t), \varphi^{\prime}(t)\right\rangle$, so $c(t)$ is integrable, and, for $t_{0} \in J$, the differential equation has a unique absolutely continuous solution $\varphi(t)=C(t) \varphi\left(t_{0}\right)$, where $C(t)=\int_{t_{0}}^{t} c\left(t^{\prime}\right) d t^{\prime} . C(t)$ is real and continuous, and $|C(t)|=1$. Therefore $C(t)=1$ and $\varphi(t)=\varphi\left(t_{0}\right)$ for $t \in J$.

4. The distinct eigenvalue condition is necessary. This is shown by an example. Let $\boldsymbol{K}_{+}$and $\boldsymbol{K}_{-}$be two square matrices, each with distinct eigenvalues, which do not commute. Define $K(t)$ as follows:

$$
\begin{aligned}
K(t) & =I+t^{2} K_{-} \quad \text { when } t \leqq 0, \\
& =I+t^{2} K_{+} \quad \text { when } t \geqq 0 .
\end{aligned}
$$

Then $K(t)$ has the following properties: 1. $K(t)$ and its eigenvalues are absolutely continuous on any finite interval, and the eigenvalues are distinct except at $t=0$. 2. $K^{\prime}(t)$ exists and $K(t) \sim K^{\prime}(t)$ at all $t$ including 0 . 3. The (continuous) eigenvectors of $K(t)$ are constant on $(-\infty, 0)$ and 
on $(0, \infty)$ and are simply the eigenvectors, respectively, of $\boldsymbol{K}_{-}$and $\boldsymbol{K}_{+}$. Thus they are not continuous across 0 . 4. If $t_{1}<0<t_{2}$, then $K\left(t_{1}\right)$ and $K\left(t_{2}\right)$ do not commute.

\section{REFERENCE}

1. A. Acker, Stability results for linear systems involving a time varying unbounded operator, Doctoral Dissertation, Boston University, 1972, Appendix B.

Department of Mathematics, Louisiana State University in New Orleans, New OrLeans, Louisiana 70122

Current address: Mathematisches Institut I, Universität Karlsruhe, 75 Karlsruhe 1, Englerstrasse 2, Germany 